\title{
Seroprevalence of the enteroaggregative Escherichia coli virulence factor dispersin among USA travellers to Cuernavaca, Mexico: a pilot study
}

Correspondence
David B. Huang
dhuang82@hotmail.com

Received 3 July 2007

Accepted 7 December 2007

\author{
David B. Huang, ${ }^{1} \dagger$ Eric L. Brown, ${ }^{2}$ Herbert L. DuPont, ${ }^{1,3}$ Jing Cerf, ${ }^{1}$ \\ Lily Carlin, ${ }^{4}$ Jose Flores, ${ }^{4}$ Jaime Belkind-Gerson, ${ }^{4}$ James P. Nataro ${ }^{5}$ \\ and Pablo C. Okhuysen ${ }^{1,4}$
${ }^{1}$ Department of Medicine, Baylor College of Medicine, 1 Baylor Plaza, Houston, TX 77030, USA
${ }^{2}$ University of Texas at Houston School of Public Health, 1200 Herman Pressler, Houston, TX 77030, USA
${ }^{3}$ St Luke's Episcopal Hospital, 6720 Bertner Avenue, Houston, TX 77030, USA
${ }^{4}$ University of Texas at Houston Medical School, 6431 Fannin, Houston, TX 77030, USA
${ }^{5}$ Center for Vaccine Development, University of Maryland School of Medicine, 655 W. Baltimore Street, Baltimore, MD 21201, USA

\begin{abstract}
This pilot study examined the change in the seroprevalence of the enteroaggregative Escherichia coli (EAEC) virulence factor dispersin in USA students during a short stay in Cuernavaca, Mexico, between June and August 2004. One hundred and ninety-five students provided paired serum samples - one on arrival to Mexico (pre-serum) and a second on departure from Mexico (post-serum) after a mean stay of 19 days. Serum samples were tested for $\lg \mathrm{G}$ antibody to a recombinant purified dispersin protein by ELISA. For all travellers, with and without diarrhoea, the mean \pm SD pre-serum absorbance value (read at 450 and $570 \mathrm{~nm}$ ) was $0.340 \pm 0.212$ and the mean post-serum value was $0.513 \pm 0.316(P<0.00001)$. Both travellers who developed diarrhoea and those who did not develop diarrhoea had an increase in lgG antibody to dispersin from the time of arrival to the time of departure from Cuernavaca (diarrhoea group $0.323 \pm 0.197$ to $0.501 \pm 0.311, P<0.00001$, and the asymptomatic group $0.354 \pm 0.224$ to $0.525 \pm 0.321$, $P<0.00001$ ). The pre-serum absorbance value (read at 450 and $570 \mathrm{~nm}$ ) for $\lg \mathrm{G}$ antibody to dispersin was not associated with protection against the development of diarrhoeal illness. These results indicate that USA travellers to Mexico show seroconversion for the EAEC virulence factor dispersin. Further studies are needed to characterize in more detail the host clinical and immunological responses to the dispersin protein.
\end{abstract}

\section{INTRODUCTION}

Travellers' diarrhoea is the most common problem experienced by people visiting developing countries (Steffen, 2005). Bacterial enteropathogens are the most common cause of travellers' diarrhoea. Among the bacteria, enterotoxigenic Escherichia coli (ETEC) and enteroaggregative E. coli (EAEC) have been reported to be the most common causes of acute diarrhoea. ETEC secretes heatlabile and heat-stable enterotoxins, which elicit an intestinal secretory response and distinguish ETEC from other diarrhoeagenic E. coli. EAEC secretes a heat-stable toxin,

tPresent address: Department of Virology, 900 Ridgebury Road, G4, Ridgefield, CT 06877, USA.

Abbreviations: AP, alkaline phosphatase; EAEC, enteroaggregative Escherichia coli; ETEC, enterotoxigenic Escherichia coli. similar to ETEC, and a protein called dispersin, which are important virulence factors in the pathogenesis of EAEC.

Dispersin is a $10.2 \mathrm{kDa}$ protein that is responsible for dispersing EAEC along intestinal epithelium and facilitating penetration of mucus along the intestinal mucosa (Sheikh et al., 2002; Nishi et al., 2003; Nataro, 2005). In one laboratory, $80 \%$ of EAEC isolates were identified as carrying the aap gene encoding dispersin (Sheikh et al., 2002). It is currently unknown whether other enteropathogens also secrete dispersin. In a volunteer study, persons challenged with EAEC developed antibodies to a protein with a molecular weight similar to dispersin suggesting that this protein may be immunogenic (Nataro et al., 1995). In this pilot study, we evaluated the seroprevalence of enteric pathogens that secrete dispersin among 195 USA travellers during a short stay in Cuernavaca, Mexico. We hypothesized that travellers 
to Mexico would have an increased seroconversion rate following infection with enteric pathogens that secreted dispersin from their time of arrival to their time of departure from Mexico and that this would correlate with EAEC infection.

\section{METHODS}

Sample collection. Paired serum samples were obtained from 195 USA students within $72 \mathrm{~h}$ of arrival and prior to their departure from Cuernavaca, Mexico (mean length of stay of 19 days). All students were healthy and without diarrhoeal illness at the time of enrolment. Collected sera were stored at $-80{ }^{\circ} \mathrm{C}$ until use. Diarrhoeal illness was defined as the passage of three or more unformed stools accompanied by at least two of the following gastrointestinal symptoms: abdominal pain, cramping, faecal urgency, excessive gas, tenesmus, nausea or vomiting. Informed consent was obtained from all participating students. This study was approved by the University of Texas at Houston Health Science Center Committee for the Protection of Human Subjects.

Identification of EAEC Stool samples were submitted to a laboratory for processing in a subset of 71 students with diarrhoea. Five lactose-positive colonies were retrieved from MacConkey agar plates from each stool sample and inoculated into individual peptone stabs that were transported to the University of Texas at Houston School of Public Health for identification of EAEC. E. coli in each stool sample were tested for the presence of EAEC on the basis of a characteristic pattern of adherence to cultured HEp-2 cells (Donnenberg \& Nataro, 1995). A sample was interpreted as positive for EAEC if it showed the characteristic 'stacked-brick' aggregative appearance. EAEC strain 042 served as a positive control. E. coli strain HS served as a negative control.

Expression and purification of recombinant dispersin. Recombinant dispersin and the control protein Borrelia burgdorferi OspC (outer surface protein C) were expressed in E. coli (JM101) (Stratagene) harbouring the corresponding plasmid as recombinant His-tagged proteins as described previously (Brown et al., 2005) and were expressed without the leader sequence, as predicted using the Signal 3.0 server (Bendtsen et al., 2004). OspC, a $22 \mathrm{kDa}$ lipoprotein, was used as a control recombinant protein in this study, and was cloned and expressed as described previously (Brown et al., 2005). aap was amplified by PCR using E. coli strain 042 DNA as a template. The following oligonucleotide primers were used: forward primer $5^{\prime}$ GCGGGATCCGGTGGTAGCGGTTGGAACGC- $3^{\prime}$ and reverse primer 5'-GCGCTGCAGTCATTATTTAACCCATTCGG-3', respectively (restriction enzyme recognition sequences are underlined). The resulting PCR amplification product was ligated into the pQE30 expression vector (Qiagen) and used to transform E. coli (JM101), and protein was expressed and purified, as described previously (Brown et al., 2005). PAGE analysis of purified dispersin demonstrates the purity of the preparation (Fig. 1). Protein concentrations were determined using a bicinchoninic acid protein assay (Pierce) and stored at $-20{ }^{\circ} \mathrm{C}$ until use.

Western ligand blot analysis. Recombinant dispersin or OspC ( $4 \mu \mathrm{g}$ per well) was subjected to SDS-PAGE (using a $15 \%$ tricine gel) and examined by staining the gel with $0.05 \%$ Coomassie brilliant blue or electrotransferring the gel to a $0.45 \mu \mathrm{m}$ nitrocellulose membrane (Bio-Rad) as described previously (Brown et al., 2005). Western blot membranes were blocked for $2 \mathrm{~h}$ at room temperature in SuperBlock (Pierce) and probed with either mouse anti-dispersin $(1: 2000$ dilution), normal mouse serum (1:2000 dilution), anti- $6 \times$ His monoclonal IgG2a $(1: 10000)$ antibody (Roche Diagnostics), or with

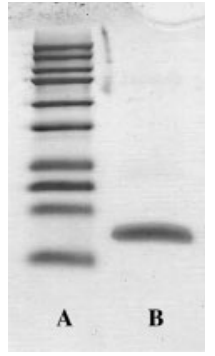

Fig. 1. Dispersin protein $(4 \mu \mathrm{g})$ was subjected to SDS-PAGE (15\% tricine gel) and stained with Coomassie brilliant blue. Lane A, molecular mass marker; lane B, N-terminal His-tagged dispersin protein.

patient serum (1:4000 dilution). The alkaline phosphatase (AP)conjugated secondary antibodies used were monoclonal goat antimouse IgG2a (1:2500 dilution; MP Biomedicals) for detection of mouse anti-dispersin and anti- $6 \times$ His antibodies, and polyclonal goat anti-human $\operatorname{IgG}\left[\mathrm{F}\left(\mathrm{ab}^{\prime}\right)_{2}\right]$ (Accurate Chemical and Scientific). All incubations were performed in $15 \mathrm{ml}$ SuperBlock for $1 \mathrm{~h}$ with shaking at room temperature and membranes were washed in TBST [0.15 M NaCl, $20 \mathrm{mM}$ Tris/HCl, $0.05 \%$ Tween 20 (USB), pH 7.4] between all steps. Membranes were probed with a monoclonal antiIgG2a AP-conjugated antibody and developed using 1-Step NBT/ BCIP (Pierce).

ELISA for the detection of IgG antibodies to dispersin. A checkerboard analysis was used to analyse antibody titres to dispersin in the serum of healthy volunteers. Serum samples with high $($ mean \pm SD $0.587 \pm 0.06)$ and low (mean \pm SD $0.045 \pm 0.06)$ absorbance values (read at 450 and $570 \mathrm{~nm}$ ) were used as positive and negative controls, respectively. Dispersin $(0.1 \mu \mathrm{g})$ diluted in $50 \mu \mathrm{l}$ sodium bicarbonate ( $\mathrm{pH} 9$ ) was added to individual wells of a Nunc flat 96-well Immulon plate (MaxiSorp ImmunoPlates) and incubated at $5{ }^{\circ} \mathrm{C}$ overnight. Each well was washed six times with PBS containing $0.1 \%$ Tween 20 between each of the steps described below. Wells were blocked with $200 \mu \mathrm{l} 5 \%$ non-fat powdered milk (Sigma) in PBS and incubated for $1 \mathrm{~h}$ at $37{ }^{\circ} \mathrm{C}$. Diluted serum $(100 \mu \mathrm{l}$ of a 1:64 dilution) in 1\% skimmed milk in PBS was added to each well in triplicate and incubated for $1 \mathrm{~h}$ at $37^{\circ} \mathrm{C}$. Polyclonal antihuman IgG raised in sheep and conjugated with horseradish peroxidase (Sigma) at 1:60000 dilution in $100 \mu \mathrm{l} 1 \%$ milk was added to each well and incubated for $1 \mathrm{~h}$ at $37^{\circ} \mathrm{C}$ as a detecting antibody. Substrate solution $(200 \mu$ l tetramethylbenzidine with hydrogen peroxide; Pharmingen) was added and incubated at room temperature in the dark for $30 \mathrm{~min}$. Stop solution $(50 \mu \mathrm{l} 1.25 \mathrm{M}$ sulfuric acid) was added and the absorbance read at 450 and $570 \mathrm{~nm}$ using a dual-length plate reader (MR5000; Dynatech). Plate-to-plate variability measurements of anti-dispersin antibodies in the positive and negative controls were standardized by ensuring that the values of the well were within 1 SD of the mean value.

Generation of antiserum. BALB/c mice (Harlan Sprague Dawley) were immunized with $20 \mu \mathrm{g}$ recombinant dispersin emulsified in complete Freund's adjuvant as described previously (Brown et al., 2005). Four weeks later, mice from each immunization group were boosted with dispersin in Freund's incomplete adjuvant and serum was collected 1 week later (Brown et al. 2005). All animal procedures were approved by the Institutional Animal Care and Use Committee of Texas A\&M University Health Science Center Institute of Biosciences and Technology (IBT), Houston, TX, USA. 
Recombinant protein purity and antiserum specificity. The purity of the recombinant dispersin used in the ELISA assays described was assessed by subjecting recombinant dispersin and the recombinant control protein OspC to SDS-PAGE electrophoresis. Coomassie brilliant blue staining (Fig. 2; lanes 1) and Western ligand blot analysis using a monoclonal anti- $6 \times$ His antibody (Fig. 2; lanes 3) revealed single dispersin and OspC bands of 13 and $22 \mathrm{kDa}$, respectively. To confirm that binding to dispersin was not a function of non-specific binding, Western ligand blot analysis using mouse antidispersin antiserum or serum from EAEC patients (Fig. 2; lanes 2 and 4, respectively) demonstrated binding to dispersin only (Fig. 2; lanes 2 and 4). Neither blots of dispersin and OspC incubated with normal mouse serum and probed with the corresponding AP-conjugated secondary antibody nor blots incubated with the secondary antibody alone revealed any colour change (data not shown).

Statistical analysis. Data were analysed by STATA. Serum IgG antibody levels to dispersin were compared using a paired, two-tailed Student's $t$-test. Seroconversion for dispersin was defined when absorbance values ( read at 450 and $570 \mathrm{~nm}$ ) taken after travel were in excess of 2 SDs above the mean baseline readings for the entire group. A $P$ value of $<0.05$ was considered significant.

\section{RESULTS}

A total of $88 / 195$ USA students (45\%) developed acute diarrhoea during their stay in Cuernavaca. The mean stay in Mexico was $19.1 \pm 7.4$ days. The mean $\pm S D$ antidispersin IgG absorbance values (read at 450 and $570 \mathrm{~nm}$ ) in pre-serum from student volunteers was $0.340 \pm 0.212$ and in post-serum was $0.513 \pm 0.316$ $(P<0.00001)$ (Fig. 3). To exclude the possibility that the observed reactivity to dispersin was due to LPS contamination, which is sometimes associated with recombinant protein purification, ELISA plate wells were coated with recombinant OspC from B. burgdorferi that was purified from the same $E$. coli strain and vector used to produce the dispersin described in this study, and tested against patient

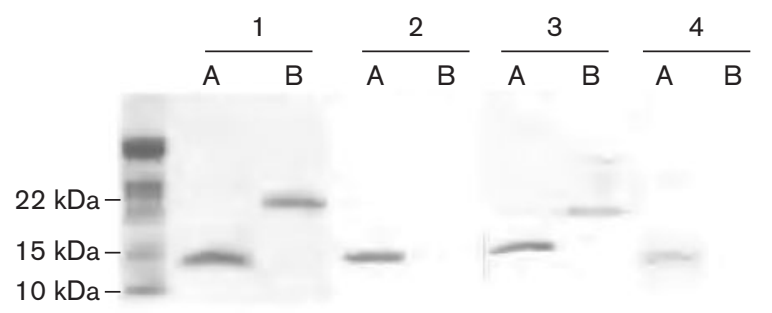

Fig. 2. SDS-PAGE and Western ligand blot analysis showing dispersin (A) and OspC (B) for each treatment group. Proteins were subjected to SDS-PAGE electrophoresis under reducing conditions ( $15 \%$ tricine gel) and either stained with Coomassie brilliant blue (lanes 1), or transferred onto nitrocellulose (lanes 24) and probed with mouse anti-dispersin polyclonal serum (lanes 2), monoclonal mouse anti-6×His (lanes 3 ) or with a representative serum sample from an EAEC patient (lanes 4). The secondary antibodies used to visualize binding are described in Methods. Blots probed with secondary antibodies alone revealed no colour change after the addition of substrate (data not shown).

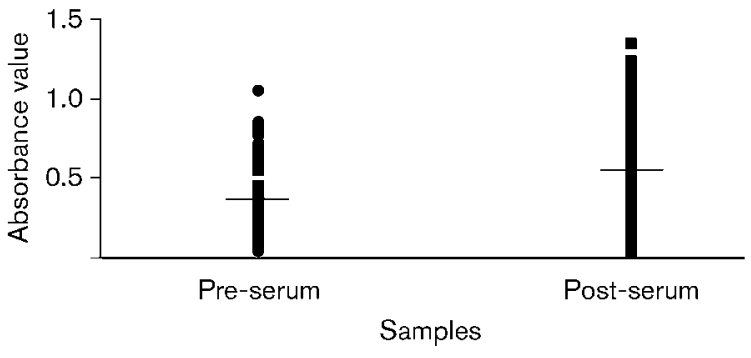

Fig. 3. Serum anti-dispersin lgG antibody seroconversion among USA students $(n=195)$ during a short stay in Cuernavaca, Mexico. Absorbance values were read at 450 and $570 \mathrm{~nm}$.

serum as described above. No IgG OspC reactivity was observed for the pre- and post-serum samples tested (data not shown). Both the students that developed diarrhoea, and those that did not, had an increase in anti-dispersin IgG at the time of departure from Cuernavaca $(P<0.00001)$ (Table 1): a total of $36 / 88$ students $(41 \%)$ of those who developed diarrhoea and 52/107 (49\%) of those who did not develop diarrhoea had an increase in anti-dispersin IgG. The pre-serum anti-dispersin IgG absorbance values were not associated with protection against the development of diarrhoeal illness. In the subset of 71 students who submitted stool samples, 5 students had EAEC identified in their stools. For these five students, the mean antidispersin IgG absorbance values in pre- and post-serum were $0.415 \pm 0.182$ and $0.952 \pm 0.381(P=0.01)$, respectively. Among the other 66 students who did not have EAEC identified in their stools, the mean anti-dispersin IgG absorbance values in pre- and post-serum were $0.352 \pm 0.216$ and $0.476 \pm 0.281(P<0.0001)$, respectively.

\section{DISCUSSION}

The objective of this study was to evaluate the rate of seroconversion for the EAEC virulence factor dispersin in a population of 195 USA travellers in Cuernavaca, Mexico,

Table 1. Serum lgG antibody to dispersin on arrival in and prior to departure from Mexico, and the development of diarrhoeal illness among USA students $(n=195)$ during a short stay in Cuernavaca

Results are shown as absorbance values (mean $\pm \mathrm{SD}$ ), read at 450 and $570 \mathrm{~nm}$.

\begin{tabular}{|lccc|}
\hline $\begin{array}{l}\text { Serum IgG antibody } \\
\text { to dispersin }\end{array}$ & $\begin{array}{c}\text { Diarrhoea } \\
(\boldsymbol{n}=\mathbf{8 8})\end{array}$ & $\begin{array}{c}\text { No diarrhoea } \\
(\boldsymbol{n}=\mathbf{1 0 7})\end{array}$ & $\boldsymbol{P}$ value \\
\hline Pre-serum & $0.323(0.197)$ & $0.354(0.224)$ & NS $(P=0.30)$ \\
Post-serum & $0.503(0.311)$ & $0.525(0.321)$ & NS $(P=0.63)$ \\
$P$ value & $P<0.00001$ & $P<0.00001$ & - \\
\hline
\end{tabular}

NS, Not significant. 
who were likely to be exposed to various enteropathogens. Our results indicated that both the travellers who developed diarrhoea and those that did not had an increase in anti-dispersin IgG antibodies at the time of departure from Mexico compared with their arrival. However, serum IgG antibodies reactive to dispersin were not predictive of protection against diarrhoeal illness when the traveller was exposed to these enteropathogens.

Approximately $68 \%$ of healthy adults in the USA have IgG antibodies to a $10.2 \mathrm{kDa}$ protein corresponding to the dispersin protein (Nataro et al., 1995). In an oral challenge study of individuals who were antibody-negative to the $10.2 \mathrm{kDa}$ EAEC protein, three out of five participants who were fed a dose of $10^{10}$ c.f.u. EAEC strain 042, the prototypical EAEC strain, developed diarrhoea (Nataro et al., 1995). All five volunteers subsequently developed an anti-dispersin secretory IgA antibody response to oral challenge with EAEC. These results suggested that dispersin is immunogenic in a human EAEC challenge model and holds considerable promise as an immunogen.

Dispersin is a low-molecular-mass $(10.2 \mathrm{kDa})$ protein secreted by EAEC. In one laboratory, $80 \%$ of EAEC strains carried the gene that encodes dispersin (Sheikh et al., 2002). This protein is responsible for 'dispersing' EAEC across the intestinal mucosa and for penetration of EAEC across the thick mucus layer above the intestinal epithelium (Sheikh et al., 2002). Dispersin is a low-molecularmass secreted protein encoded by the EAEC gene aap. This gene lies immediately upstream of aggR, the gene encoding a master regulator of a myriad of plasmid genes, including aap and chromosomal genes that express virulence factors. Dispersin requires an ATP-binding cassette complex transporter, encoded by the gene aatA, for outer-membrane translocation where it non-covalently attaches to the surface of the bacterium (Sheikh et al., 2002; Nishi et al., 2003).

Limitations exist in this pilot study. The serum samples for IgG antibody to dispersin were not measured at the same time point for all subjects. Students provided paired serum samples, one on arrival in Mexico (pre-serum) and the second on departure from Mexico (post-serum) after a mean of 19 days (range 9-40 days). Also, considering the delay from acquisition of enteric infection until mounting a humoral immune response, the study would be improved by obtaining a serum sample 7-10 days after leaving Mexico. Optimal antigen concentration and antibody titre to dispersin was determined by a checkerboard analysis. Anti-dispersin IgG antibody titres were not performed beyond the 1:64 dilution. We were unable to screen for secretory IgA reactive to dispersin. This isotype may be important in host clinical and immunological responses to dispersin, as it is involved in protection against infections or disease severity resulting from EAEC infections. Since we were unable to correlate anti-dispersin IgG responses with protection or susceptibility to travellers' diarrhoea, future work will evaluate the seroconversion rate of participants for the EAEC virulence factor, dispersin, in the context of IgG, secretory IgA and IgM reactivity to dispersin in samples from convalescent serum and faeces samples collected 7-10 days after the traveller leaving Mexico.

In conclusion, this pilot study determined the seroconversion rate of USA students travelling to Cuernavaca, Mexico, for the EAEC virulence factor dispersin to be high in both travellers who developed diarrhoea and those who did not develop diarrhoea. Acknowledging the limitations of this study, the high seroconversion rate suggests that EAEC infections are common in both symptomatic and asymptomatic travellers to Mexico. More studies are needed to evaluate the seroconversion status among individuals infected with EAEC, and to characterize in more detail the host clinical and immunological responses to the EAEC virulence factor dispersin.

\section{ACKNOWLEDGEMENTS}

We thank Dr Gabriela Bowden (Texas A\&M Institute of Biosciences and Technology) for her generosity in providing the mice and the facilities required to perform the vaccinations. We would also like to thank the participating students and the Universidad Internacional in Cuernavaca, Mexico, for participating in this study. This study was financially supported by the National Institutes of Health (grant 1 K23 AI6439-01 to D. B. H.).

\section{REFERENCES}

Bendtsen, J. D., Nielsen, H., von Heijne, G. \& Brunak, S. (2004). Improved prediction of signal peptides: Signal P 3.0. J Mol Biol 340, 783-795.

Brown, E. L., Reisenbichler, E. S., Kim, J. H. \& Höök, M. (2005). Multicomponent Lyme vaccine: three is not a crowd. Vaccine 23, 3687-3696.

Donnenberg, M. S. \& Nataro, J. P. (1995). Methods for studying adhesion of diarrheagenic Escherichia coli. Methods Enzymol 253, 324-336.

Nataro, J. P. (2005). Enteroaggregative Escherichia coli pathogenesis. Curr Opin Gastroenterol 21, 4-8.

Nataro, J. P., Deng, Y., Cookson, S., Cravioto, A., Savarino, S. J., Guers, L. D., Levine, M. M. \& Tacket, C. O. (1995). Heterogeneity of enteroaggregative Escherichia coli virulence demonstrated in volunteers. J Infect Dis 171, 465-468.

Nishi, J., Sheikh, J., Mizuguchi, K., Luisi, B., Burland, V., Boutin, A., Rose, D. J., Blattner, F. R. \& Nataro, J. P. (2003). The export of coat protein from enteroaggregative Escherichia coli by a specific ATPbinding cassette transporter system. J Biol Chem 278, 45680-45689.

Sheikh, J., Czeczulin, J. R., Harrington, S., Hicks, S., Henderson, I. R., Le Bouguenec, C., Gounon, P., Phillips, A. \& Nataro, J. P. (2002). A novel dispersin protein in enteroaggregative Escherichia coli. J Clin Invest 110, 1329-1337.

Steffen, R. (2005). Epidemiology of traveler's diarrhea. Clin Infect Dis 41, S536-S540. 